\title{
Le théâtre romain de Lillebonne. Etude des sources et nouvelle campagne de fouilles
}

\section{Vincenzo Mutarelli}

\section{(2) OpenEdition \\ 1 Journals}

Édition électronique

URL : http://journals.openedition.org/edl/117

DOI : $10.4000 /$ edl. 117

ISSN : 2296-5084

Éditeur

Université de Lausanne

\section{Édition imprimée}

Date de publication : 15 mai 2011

Pagination : 223-262

ISBN : 978-2-940331-25-3

ISSN : 0014-2026

Référence électronique

Vincenzo Mutarelli, «Le théâtre romain de Lillebonne. Etude des sources et nouvelle campagne de fouilles », Études de lettres [En ligne], 1-2 | 2011, mis en ligne le 15 mai 2014, consulté le 19 décembre 2020. URL : http://journals.openedition.org/edl/117 ; DOI : https://doi.org/10.4000/edl.117 


\section{LE THÉÂTRE ROMAIN DE LILLEBONNE ÉTUDE DES SOURCES ET NOUVELLE CAMPAGNE DE FOUILLES}

Le théâtre romain de Lillebonne a été identifié et publié en 1764 par le comte de Caylus. Redécouvert par François Rever en 1812, il est acquis par le Conseil général de la Seine-Maritime en 1818. Ses structures, constituées de substructions concentriques et radiales, sont dégagées entre 1822 et 1840, date à laquelle l'édifice est inscrit à l'inventaire des Monuments historiques. Après une période d'abandon, des fouilles complémentaires ont lieu de 1908 à 1915, puis de 1935 à 1939. Le théâtre présente la particularité d'associer une arène et un bâtiment de scène de grandes dimensions. Jusqu'à aujourd'hui, les trois époques de construction identifiées dans ses structures n'avaient jamais pu être datées. La dernière campagne de fouilles de 2007-2009 dans la partie est permet de comprendre l'évolution de l'édifice dans ses deux derniers états et de dater leur construction, ainsi que l'abandon du monument de spectacle et sa transformation en forteresse.

La ville normande de Lillebonne, située à proximité de l'embouchure de la Seine, a succédé à la ville romaine de Juliobona, capitale de la civitas du peuple des Calètes, sur les ruines de laquelle elle a été construite.

Au lendemain de la pacification de la Gaule, les Caleti choisissent d'implanter leur capitale Juliobona sur un nœud de communication stratégique pour le commerce à destination de la Britannia, du centre de la Gaule par la Seine, ou vers les peuples du Sud par sa position sur le dernier passage de traversée du fleuve. La qualité des vestiges antiques retrouvés sur le site, plus ou moins fortuitement, lors de travaux urbains, est révélatrice de l'importance et de la prospérité de la ville pendant les trois premiers siècles de notre ère. 
Fig. 1 - Vue aérienne du théâtre de Lillebonne, vers 1995.

Le théâtre romain de Lillebonne témoigne de cette grandeur. Ce monument, redécouvert au début du XIX ${ }^{\mathrm{e}}$ siècle, a connu de multiples vicissitudes historiques et architecturales qui rendent son analyse et l'établissement de sa chronologie particulièrement complexes. Pourtant, son importance a été relevée par tous ceux qui s'y sont intéressés, tant d'un point de vue monumental, puisqu'il s'agit de l'un des édifices antiques de spectacle les plus étendus et les mieux conservés du Nord de la France, que typologique.

En effet, une première confrontation avec les nombreux théâtres antiques identifiés sur le territoire de la Gaule et sur l'ensemble de l'Empire romain tout entier permet d'y déceler des particularités dont on trouve peu d'autres exemples. L'étude exhaustive de ces caractéristiques typologiques pourrait permettre de préciser la spécificité des spectacles 
qui s’y déroulaient et de contribuer à un débat déjà riche sur la pratique et le goût des spectacles en Gaule romaine.

Dans l'état actuel des connaissances archéologiques, le théâtre de Lillebonne présente trois périodes de construction que révèlent les structures apparentes encore en élévation, mais dont aucune n’avait jamais pu jusqu'ici être datée avec précision.

Les ruines du théâtre de Lillebonne telles qu'elles se présentent aujourd'hui sont le résultat de deux siècles de fouilles, d'interventions, d'entretiens, d'abandons et de restaurations successifs qui ont considérablement modifié les structures initiales telles qu'elles ont été mises au jour (fig. 1). La compréhension du monument et de son histoire passe donc nécessairement par une analyse fine de toutes les sources accessibles afin de reconstituer les indices aujourd'hui disparus, mais essentiels pour interpréter l'ensemble des données encore disponibles sur le terrain. L'analyse de l'existant, visible ou encore à découvrir, ne peut être menée correctement qu'en la complétant par ce qui a été vu et détruit au cours des années, moyen indispensable pour approcher la réalité de ce monument.

\section{La Roquette: le site du théâtre jusqu'au XVIII siècle}

\subsection{Chroniques de l'abbaye de Saint-Wandrille}

La première allusion écrite qui semble concerner en partie les ruines du théâtre antique de Lillebonne apparaît dans les Chroniques de l'abbaye de Saint-Wandrille ${ }^{1}$. Celles-ci relatent la construction de l'abbatiale, au VIII ${ }^{\mathrm{e}}$ siècle, qui fut l'occasion de la récupération de matériaux sur le site du castrum de Lillebonne, qui englobait les structures du théâtre antique:

Erinhardus aedificavit basilicam beatissimi Michaelis Archangeli, licet modico, pulcherrimo tamen aedificio allatis videlicet Petris Juliobona castro quondam nobilissimo ac firmissimo.

I. Cronicae Fontanellensis Abbatiae, p. 129. 
1.2. Premières mentions médiévales et modernes

Au Moyen Age, le site apparaît dans les textes sous le nom de «la Roque», puis de «la Roquette». L'attestation ancienne de ces deux noms semble pouvoir trouver son origine dans un terme emprunté au latin vulgaire, rocca, qui identifie un lieu fortifié élevé, plutôt que dans le mot latin classique rupes («roche»). Il aurait désigné très tôt le site du théâtre, transformé pour la défense de la ville en place fortifiée avancée, nous renseignant ainsi sur certaines caractéristiques essentielles de l'histoire du monument et de ses structures.

Jusqu'à la Révolution française, le terrain appartient au domaine non fieffé du comté de Lillebonne: il est transformé en jardin et verger, et baillé à ferme depuis le Moyen Age jusqu’à sa vente comme bien national.

Dès le XIVe siècle, plusieurs comptes de la seigneurie de Lillebonne le mentionnent. Le 10 décembre 1880, le vicomte d'Estaintot rapporte devant la Commission des Antiquités de la Seine-Inférieure les allusions au site du théâtre qu'il a pu relever dans les archives ${ }^{2}$. Elles fournissent quelques informations sur son état à la fin du Moyen Age, puis au cours des siècles suivants.

Il apparaît dans un compte de la seigneurie de Lillebonne pour les années 1359, 1374, 1384, 1388, 1395, etc. avec des changements d'attributaire et de montant de la rente:

De la ferme de l'erbage et fritage du gardin de la Roque que tient Jehan Le Mongnier au terme St Michel 1359 pour la moitié tiers sixte... 38s. $6 \mathrm{~d}$.

A partir de 1478, le site est mentionné dans les textes sous le nom de «la Roquette»:

Le jardin de la Roquette assis au dessous du castel adjugé à Jehan Le Fèvre, $20 \mathrm{~s}$.

En 1553, les bornes du terrain sont précisées:

2. R. Langlois, vicomte d'Estaintot, Procès verbaux de la Commission des Antiquités de la Seine-Inférieure. Dans son article, le vicomte d'Estaintot ne cite pas les cotes des documents qu'il a consultés. 
La ferme de la Roquette, ainsy qu'elle contient et estend, bournée d'un côté le domaine du sieur du Toupin, d'autre côté le chemin tendant du bout de la Houssaye à l'hospital de Lillebonne, d'un bout le chemin tendant du Mesnil audit bois de Lillebonne, et d'autre bout, Gilles le Seigneur et Jehan Vasse. Elle a été baillée et adjugée à Pierre de la Haye, sergent, par le prix et somme de 20 st. 6 livres de lin.

Quelques années plus tard, la description qui en est faite nous informe de manière plus détaillée sur ce qu'il reste du théâtre à cette époque:

Item en icelluy lieu, assez pres du chasteau, y a ung lieu nommé la Roquette, euquel y a grand apparence d'ediffices, iceulx assis sur un petit costeau en pendant. Y a bassecourt et belle apparence de porte, et au bout d'icelles y a encore apparence de murailles qui sont encore en evidence, et les autres sont tombées à terre.

Au XVI ${ }^{\mathrm{e}}$ siècle, il semble donc qu'une porte d'entrée du théâtre de «belle apparence» était encore en élévation.

Plus tardivement, l'Abbé Cochet cite dans La Normandie souterraine ${ }^{3}$ l'extrait d'un contrat en date du 21 août 1701 :

Un héritage planté d'arbres fruitiers, édifié de fortifications tombant en ruines et une pièce de terre labourable, nommée la Roquette, sise en ladite paroisse, contenant ensemble cinq vergées ou environ, bornée d'un côté par le sieur Dumanoir, de l'autre par les héritiers du sieur Dehors, d'un bout la rue tendant du Val-Infray à Lillebonne, de l'autre le sieur Dumanoir.

En revanche, la description de Lillebonne publiée par Dom Toussaint Duplessis en 1740 ne fait aucune allusion au théâtre, et encore moins à son origine antique ${ }^{4}$. Il faut attendre la seconde moitié du XVIII ${ }^{\mathrm{e}}$ siècle pour que le comte de Caylus identifie sur le site de la Roquette la présence d'un théâtre antique.

3. Abbé J. B. D. Cochet, La Normandie souterraine, p. 100.

4. Dom T. Duplessis, Description géographique et historique de la Haute-Normandie, tome 1, p. 3-7. 
1.3. Identification par Duchesne et le comte de Caylus

Les prémisses du renouveau de l'intérêt pour le site antique de Lillebonne et pour son théâtre en particulier datent du milieu du XVIII ${ }^{\mathrm{e}}$ siècle avec un première identification par l'ingénieur des ponts et chaussées Duchesne qui, comme beaucoup de ses collègues chargés d'améliorer le réseau des routes sur l'ensemble du territoire français, informe à sa demande le comte de Caylus, éminent antiquaire, des structures antiques qu'il rencontre et identifie sur son chemin.

En 1763, le comte de Caylus publie un relevé du site dans son Recueil d'Antiquités égyptiennes, étrusques, grecques, romaines et gauloises ${ }^{5}$ (fig. 2). La diffusion de ces informations ne devait pas avoir de conséquences immédiates sur le site.

\section{Historique des fouilles et analyse des méthodes (1812-2000)}

Tout au long du XIX siècle, les opérations archéologiques qui se déroulent dans le théâtre suivent les mêmes modes de fonctionnement: déblaiement des structures pour dégager le monument et permettre une vision d'ensemble, évacuation des terres et des débris, conservation des pierres sculptées et des objets, vente des pierres et des moellons non décorés, qui produit un complément de budget pour la suite des travaux.

Les responsables officiels des chantiers, François Rever, Emmanuel Gaillard, Achille Deville, puis par la suite Léon de Vesly, ne sont pas présents de manière continue sur le terrain. Ils supervisent de loin les travaux effectués par des intermédiaires locaux, qui n'ont pas de compétence particulière.

C'est dans les rapports au jour le jour que l'on trouve les renseignements les plus précieux et les plus précis sur les informations trouvées en place. Plus que les interprétations des responsables, ce sont donc ces comptes rendus qui doivent être analysés avec minutie pour relever les indices utiles à la reconstitution des données.

5. A. C. Ph. de Caylus, Recueil d'Antiquités égyptiennes, étrusques, grecques, romaines et gauloises, VI, p. 394-396, pl. CXXVII. 
Fig. 2 - Plan du château et du théâtre de Lillebonne (comte de Caylus, Recueil d'Antiquités, 1764, pl. CXXVI, d'après le dessin communiqué par M. Duchesne). 


\subsection{François Rever, $1812-1826$}

La Révolution et la redistribution des biens qu'elle entraîne, associée à l'essor industriel qui sort le bourg de Lillebonne de sa léthargie pluriséculaire, attirent enfin l'attention locale sur les ruines qui émergent alors à peine du sol. La terre de La Roquette, propriété de la famille d'Harcourt depuis plusieurs siècles est vendue comme bien national et rachetée par Pierre Caron, cafetier à Lillebonne. Celui-ci souhaite aplanir son terrain pour planter une pépinière dans la partie nord-est: il vide alors les remblais et les matériaux du cuneus $\mathrm{C} 10$ qui sont évacués et vendus.

En 1812, la découverte d'un trésor monétaire alerte François Rever qui établit le rapprochement avec le site identifié par le comte de Caylus dans son Recueil d'Antiquités. François Rever suit de près les excavations auxquelles procède Pierre Caron et les destructions qui s'ensuivent.

Après le démantèlement et la vente de l'escalier nord-est, Pierre Caron porte ses interventions dans la partie sud-ouest, à l'emplacement de la galerie périphérique qu'il commence à libérer de ses terres. Un premier vomitorium, le vomitorium V8, est alors déblayé, ses marches démontées et vendues. François Rever en obtient un relevé précis avant sa destruction.

François Rever est alors convaincu d'avoir affaire à un théâtre antique. Il essaie d'en déterminer le plan d'ensemble à partir de ce qui est alors visible. Il est persuadé de l'importance du site qu'il tente de sauver en attirant l'attention de l'Académie de Rouen et du Conseil général de la Seine-Inférieure. Il achète à Pierre Caron le droit d'effectuer une tranchée à l'emplacement de l'arène jusqu'à une profondeur de 2,60 m environ (8 pieds) sans que le sol primitif ne soit atteint.

Le site du théâtre est acquis par le Conseil général de la SeineInférieure en 1818. Cet achat va déterminer un changement dans les buts d'exploitation du site, mais assez peu dans les méthodes. François Rever, curé assermenté, puis député de l'Eure à l'Assemblée législative, passionné d'archéologie, est choisi par la Commission des Antiquités pour superviser les fouilles officielles financées par le département. Il a pour objectif assigné un déblaiement partiel du théâtre, qui permette une vision de la totalité en limitant les frais. Les résultats de la première campagne seront déterminants pour la poursuite éventuelle des opérations.

Une tranche du théâtre doit donc servir d'échantillon: une travée de la cavea entre deux vomitoires, pour vérifier la présence d'un passage de 
Fig. 3 - Plan horizontal de l'ancien théâtre de Lillebonne par M. Videbout, 1819, AD76, 4 T 263. 
circulation au milieu des gradins, le vomitoire lui-même et l'existence d'accès à des couloirs souterrains reliant les vomitoires entre eux, enfin l'accès des vomitoires à la galerie périphérique jusqu'au sol dallé de celleci (fig. 3). La fouille doit permettre aussi de vérifier la hauteur totale des murs subsistants en atteignant les fondations.

La présence de François Rever sur le site n'est qu'épisodique. Quelques ouvriers sont employés au déblaiement sous le contrôle du juge de paix de Lillebonne, Michel Lechaptois, qui lui communique les résultats. Les méthodes se limitent au déblaiement des terres et à la conservation des objets qu'elles contiennent.

François Rever résume le résultat des fouilles et ses interprétations dans son Mémoire sur les ruines de Lillebonne ${ }^{6}$.

2.2. Eloi Pigné, témoin des fouilles, 1812-1831

Charles Basile Eloi Pigné, pharmacien à Lillebonne et "historien indigène», a laissé un mémoire sur la ville et les antiquités de Lillebonne rédigé en 1831, qu'il intitule Panorama de Lillebonne. La quatrième partie de cette étude a pour titre "Archéologie ou Notice sur les antiquités de Lillebonne». L'abbé Cochet y voit une source essentielle pour l'étude de Lillebonne antique ${ }^{7}$.

Au paragraphe 3, dans lequel il aborde le théâtre après avoir disserté sur le castellum et l'aqueduc, il fait une synthèse des premières fouilles effectuées dans le théâtre par François Rever, puis Emmanuel Gaillard, qu'il ne nomme pas, et de la découverte progressive des structures, ce qui permet d'en préciser l'historique. Son témoignage est plus documenté que celui de François Rever en raison de sa résidence permanente à Lillebonne et de l'intérêt qu'il prend au suivi des découvertes. Il collabore à l'étude des vestiges, puisque c'est lui qui dessine l'escalier trouvé dans le vomitorium V7 à l'ouest du théâtre, à l'intention de François Rever $^{8}$, avant son démontage et la vente de ses matériaux par Pierre Caron.

6. F. Rever, Mémoire sur les ruines de Lillebonne, arrondissement du Havre, SeineInférieure.

7. Abbé J. B. D. Cochet, La Normandie souterraine, p. 105, note 3.

8. F. Rever, Mémoire sur les ruines de Lillebonne, arrondissement du Havre, SeineInférieure, pl. 3. 
La position d'Eloi Pigné, dans le contexte général du chantier, n'est pas très claire. Il prend à ferme pour six ans en 1821 une partie du terrain du théâtre encore planté d'arbres fruitiers. Il ne paiera jamais le montant de son fermage au Conseil général, protestant que les travaux incessants qui se déroulent sur le terrain ne lui permettent pas d'en avoir l'usage. Il transfère son droit de location à Michel Lechaptois, juge de paix, auquel il est apparenté.

Eloi Pigné décrit précisément la stratigraphie présente dans la galerie périphérique, entre les accès des deux vomitoires V8 et V7 (fig. 4):

Dans cette partie, on trouva divers morceaux d'antiquités, des médailles grandes et petites, des vases, des pierres gravées, des épingles d'os, des dents de sanglier, des bagues et des figurines en bronze. [...] tout ce qu'on trouve de curieux, se rencontre toujours dans une couche de terre noire mêlée de cendres et de charbon, ce qui annonce que le monument a été la proie des flammes.

Eloi Pigné signale l'identification dans les murs de la galerie circulaire de plusieurs accès obstrués à la hâte à une période tardive avec des pierres de réemploi en grand appareil. La première se trouve en face de l'escalier du vomitorium V7. L'embrasure de l'escalier est elle-même «murée sans mortier par une pile de pierres dures quarrées".

Au cours du déblai de la galerie circulaire, d'autres entrées murées sont identifiées dans les deux murs concentriques, extérieur et intérieur. Eloi Pigné signale cependant comme la plus remarquable celle qui bouche «la grande entrée du côté de l'est, au bas de la muraille extérieure du théâtre", qui sera démontée au XX $\mathrm{XX}^{\mathrm{e}}$ siècle. Les fouilles effectuées par Monsieur Renault dans le terrain de l'ancien cimetière de l'Hôpital révèlent que la grande entrée ouest présente un système de clôture identique ${ }^{9}$.

\subsection{Emmanuel Gaillard, 1827-1836}

Emmanuel Gaillard remplace François Rever dans la surveillance des travaux de déblaiement. En dix ans, il mène à bien le dégagement presque complet des structures. La galerie périphérique est totalement dégagée, puis il fait fouiller les structures de la cavea, pour terminer par l'arène.

9. E. Pigné, Panorama de Lillebonne, p. 343. 
Fig. 4 - Statuette en bronze, II ${ }^{\mathrm{e}}$ siècle apr. J.-C., retrouvée dans les remblais de fouilles du XIXe siècle. 
Par sa résidence au château de Folleville, village dont il est maire, Emmanuel Gaillard est voisin du site du théâtre, mais ses méthodes restent les mêmes: c'est maintenant le maire de Lillebonne, François Herbet, qui dirige les ouvriers et suit les opérations de terrain sous son contrôle. Il lui rend compte par écrit de l'avancement des travaux.

Emmanuel Gaillard fait une synthèse dans trois dissertations restées inédites qu'il remet à la Commission des Antiquités et à l'Académie des Inscriptions et Belles-Lettres ${ }^{10}$. Il cherche à analyser les découvertes apportées par la fouille du théâtre, de manière plutôt théorique. Pour son interprétation, il s'appuie sur la publication récente du théâtre d'Herculanum par François Mazois ${ }^{11}$ : il établit une correspondance exacte entre ce théâtre et ce qu'il trouve à Lillebonne. Il interprète ce qu'il découvre en référence à ce modèle et oriente les travaux à venir en fonction de ses conclusions.

Comme à Herculanum, il place le podium ou loge officielle du tribunal à l'extrémité est de la cavea, à l'emplacement du premier vomitorium. Continuant le parallèle, il croit découvrir la scène à l'emplacement de l'arène entre les deux entrées axiales, mais il n'y rencontre que le vide, ce qui ne manque pas de l'étonner. Il ne sait pas comment interpréter les structures des podia du nord-est et du nord-ouest, qu'il imagine se trouver en arrière de la scène. Il fouille également les thermes de l'arène qu'il pense faire partie des bâtiments de scène du théâtre et de pièces de service pour les acteurs.

Dans ses descriptions, il relève parfois des éléments stratigraphiques permettant d'établir une chronologie de construction, mais ses conclusions sont souvent erronées. Il reconnaît l'existence de plusieurs états du théâtre et sa transformation en forteresse à une époque tardive. Mais il n'hésite pas à vider le cuneus $\mathrm{C} 5$ de ses remblais, car il pense que l'apport des terres qu'il contient date de l'utilisation défensive postérieure; il n'y voit donc pas une composante structurelle du dernier état du théâtre. Il prend cependant fermement position contre le projet de François Herbet et du préfet de vider entièrement les remblais du cuneus C8: pour retrouver l'état antérieur de cet espace autrefois ouvert sur la galerie périphérique, l'aspect du dernier état de l'hémicycle de la cavea sera

Io. E. Gaillard, Trois dissertations sur diverses parties du théâtre romain de Lillebonne.

II. F. Mazois, Les Ruines de Pompéi et "Considérations sur la forme et la distribution des théâtres antiques". 
Fig. 5 - Plan de la ville de Lillebonne par M. Dumas, 1846. Lillebonne, cadastre. 
définitivement perdu, entraînant un contresens archéologique. Il nous apprend ainsi qu'avant d'être en partie vidé, le cuneus C8 présentait dans sa partie haute les mêmes murs «formant loges" que le cuneus $\mathrm{C} 1$.

Emmanuel Gaillard meurt brusquement en 1836, sans avoir terminé le déblaiement du théâtre.

\subsection{Achille Deville, 1836-1841}

Nouveau directeur du Musée des Antiquités de la Seine-Inférieure, récemment créé pour accueillir une partie des vestiges trouvés à Lillebonne, Achille Deville remplace Emmanuel Gaillard. Il suit les dernières opérations jusqu'en 1841. Les sources sont plus rares pour cette période et se limitent souvent aux comptes rendus imprimés dans le Bulletin de la commission départementale des antiquités.

Un rapport rédigé par Achille Deville en 1836 mentionne cependant les dernières trouvailles faites dans la périphérie immédiate du théâtre à l'ouest : il propose de les rattacher aux structures de défense construites à l'époque tardive ${ }^{12}$. Les murs qui les composent sont constitués de pierres de réemploi, souvent sculptées, provenant de monuments funéraires ou d'autres monuments abandonnés par suite des destructions.

Achille Deville prend soin de décrire le mobilier extrait des ruines et rassemblé dans le Musée des antiquités dont il a la charge, qui compose alors l'essentiel des collections.

Le monument de spectacle antique de Lillebonne est l'un des premiers à être classé à l'inventaire des Monuments historiques, dès 1840 (fig. 5).

La deuxième moitié du XIXe siècle est marquée par un abandon du monument. Le déblaiement est alors à peu près terminé, à l'exception de la scène que sa situation sous la route départementale rend inaccessible (fig. 6). La Commission des Antiquités s'intéresse dorénavant à d'autres sites régionaux et l'abbé Cochet, inspecteur des monuments historiques et responsable de la conservation du théâtre, préfère focaliser ses recherches sur les sites funéraires. En 1874, ce dernier autorise la destruction d'un mur du théâtre à l'ouest, parce qu'il gêne les résidents voisins des ruines.

A la fin du siècle, le théâtre a beaucoup souffert de l'abandon. Un mur de clôture en métal destiné à l'isoler et à le protéger des dégradations

I2. A. Deville, Notes sur les sculptures et les mosä̈ques découvertes à Lillebonne en 1836. 
Fig. 6 - Lillebonne vers 1850 , vue prise du haut du théâtre, après la construction de la mairie, lithographie extraite de La Normandie illustrée.

et du dépôt d'immondices est finalement édifié sur tout son périmètre. Des travaux de restauration ponctuels sont effectués pour consolider les murs.

\subsection{Léon de Vesly, 1906-1915}

Au début du $\mathrm{XX}^{\mathrm{e}}$ siècle, Léon de Vesly, nouveau directeur du Musée des Antiquités de Seine-Inférieure, tente de reprendre en main la gestion du théâtre. De 1908 à 1915, il fait procéder à des campagnes de fouilles successives, sous le contrôle du gardien du théâtre André Guyot qui l'informe de l'évolution des travaux par des rapports réguliers et circonstanciés.

Plusieurs zones sont plus particulièrement l'objet de ses explorations: l'entrée axiale ouest qui peut enfin être fouillée après l'acquisition du terrain par le département le long de la rue du Toupin et ses accès extérieurs, l'entrée axiale est et la galerie périphérique est, le pourtour de l'arène, l'escalier nord-ouest.

Plus que les articles publiés par Léon de Vesly pour rendre compte des dernières trouvailles faites sur le site, dont les résultats sont passés au crible de ses interprétations parfois discutables, les comptes rendus des 
Fig. 7 - Le mur de fermeture de l'entrée est avant son démontage par Paul Franchette en 1938.

fouilles du gardien André Guyot, documentés par de nombreux schémas, sont une source de renseignements irremplaçables sur la stratigraphie des zones explorées ${ }^{13}$.

André Guyot est à la recherche de circulations souterraines qui pourraient exister sous les structures de la cavea. Il localise ainsi certains accès à des galeries aujourd'hui invisibles.

La guerre de 1914 met fin provisoirement aux campagnes de fouilles dans le monument.

\subsection{Paul Franchette, $1935-1938$}

De 1935 à 1938, Paul Franchette, architecte en chef des monuments historiques, suit sous la direction d'Albert Grenier, professeur au collège de France, les travaux menés dans le théâtre de Lillebonne par l'entreprise Lanfry. Des sondages sont réalisés dans de nombreuses parties du monument pour permettre à Albert Grenier de répondre aux questions qu'il se pose sur la typologie du monument. Il fera des interprétations

I3. A. Guyot, dossiers «Lillebonne, travaux et découvertes». 
issues de ces investigations un chapitre de son Manuel d'archéologie galloromaine $^{14}$.

En 1935, suivant les indications d'Albert Grenier, Paul Franchette ouvre une tranchée dans la cavea pour vérifier l'existence de gradins de pierre. N'en trouvant pas les vestiges de fondation dans le gros œuvre, il conclut à une cavea constituée de remblais recouverts de gazon et de gradins de charpenterie.

En 1936, il s'intéresse à l'arène et creuse une travée au centre selon le petit axe pour obtenir des informations sur la typologie du monument: amphithéâtre complet ou demi-amphithéâtre. Deux galeries creusées à douze mètres sous la place Félix Faure n'offrent aucun résultat. Une troisième permet de dégager un mur orienté parallèlement au grand axe de l'arène. Il effectue des sondages sur le pourtour de l'arène pour étudier les maçonneries qui la cernent (fig. 8 et 9).

La même année, Paul Franchette réalise une fouille vers l'entrée axiale est et retrouve les fondations du piédroit à l'entrée du vomitorium V1 qu'il décide de laisser apparent.

Les sondages se poursuivent, en 1937, au pied du podium pour en étudier les fondations. Il pense identifier l'assise de la maçonnerie du proscaenium au pied du talus en contrebas de la place.

Le programme établi pour 1937 par Albert Grenier prévoit également le dégagement de la cage de l'escalier situé à l'est de la scène (E1), complètement remblayé. Après l'enlèvement des terres de la partie supérieure, Paul Franchette remarque que le parement y est encore en bon état ${ }^{15}$.

En 1938, il poursuit le déblaiement de la cage d'escalier est (E1) totalement récupéré en 1812. Il détruit ainsi définitivement les remblais structurels (aggestus) qui soutenaient les marches: il reconnaît «les parties basses des murs ayant constitué la partie antérieure de la cage» ${ }^{16}$.

La structure qui ferme l'entrée axiale orientale est démontée pour retrouver les blocs moulurés (fig. 7 et 8 bis). Des sondages peu étendus mais profonds sont réalisés au niveau de l'entrée extérieure vers le vomitorium $\mathrm{V} 3$.

I4. A. Grenier, Manuel d'archéologie préhistorique, celtique et gallo-romaine.

I5. P. Franchette, Rapport sur les fouilles archéologiques exécutées de 1935 à 1937 au théatre antique de Lillebonne...

16. P. Franchette, Rapport sur les fouilles archéologiques et travaux exécutés en 1938. 
Fig. 8 - Fouilles de l'arène par Paul Franchette, années 1935-1938.

Fig. 8bis — Démontage du mur de fermeture de l'entrée axiale est en 1938. 
Fig. 9 - Fouilles de l'arène par Paul Franchette, années 1935-1938. 
Les recherches archéologiques dans le théâtre de Lillebonne sont interrompues en 1939 par le nouveau conflit mondial. Le monument antique trouve alors une utilisation inattendue. Pendant toute la durée de la guerre, il sert en effet de mausolée à l'officier allemand commandant la place de Lillebonne mort en 1940. Sa sépulture est placée au pied du podium, à l'emplacement du sacellum. La dépouille sera évacuée par les armées d'occupation en 1944.

\subsection{Maurice Yvart, $1960-1962$}

En 1960, après vingt ans d'abandon, le développement de la végétation sur les structures du théâtre nécessite d'urgence une intervention. La mairie de Lillebonne souhaitant mettre en place une illumination de ses monuments, des spots d'éclairage alimentés par des câbles électriques sont alors installés dans tout le parcours de la galerie à l'est. L'inauguration de ces illuminations a lieu le 19 juillet 1960.

Les premières interventions dans la galerie est par Maurice Yvart, pharmacien à Lillebonne et correspondant local de la Commission des Antiquités, datent de la mise en place du réseau électrique. Elles se poursuivent en 1961 et 1962 par une campagne de restauration qui intéresse en particulier la galerie périphérique ouest et celle de l'est entre les vomitoria V1 et V2. Il recouvre une partie des vestiges fouillés, à l'entrée est en particulier, au moyen de gravats d'origines diverses pour aplanir le terrain.

\subsection{Les travaux de Georges Duval en 1974}

A partir de 1974, une nouvelle campagne de restauration est programmée sous la direction de l'architecte en chef des monuments historiques Georges Duval. Lors de cette campagne, de nombreuses parties du monument font l'objet de restaurations des parements et des noyaux, là où les dégradations étaient très importantes. Les fouilles associées à ces restaurations sont superficielles et situées à proximité immédiate des murs, elles se sont donc superposées à celles des années 1960-1962. 
Relevé topographique des vestiges du théâtre de Lillebonne en 2009. 


\section{Description}

\subsection{Présentation des structures}

Aujourd'hui, l'édifice présente la composition suivante: une galerie périphérique qui gère les accès et la distribution interne, l'hémicycle de la cavea et une arène, auxquels il convient d'ajouter le bâtiment de scène encore enfoui sous la route départementale et la place Félix Faure.

Trois types de structures portantes peuvent être identifiés: les substructions concentriques, présentes essentiellement dans la galerie périphérique et dans l'arène, les substructions rayonnantes de la cavea, les remblais structurels artificiels (aggestus).

La configuration actuelle du théâtre offre une géométrie complexe, conçue pour s'adapter aux structures préexistantes tout en s'intégrant dans l'urbanisme de la ville antique. L'ima cavea suit la forme ellipsoïdale de l'arène. La summa cavea, parfaitement circulaire au sud, devient rectiligne à l'est et à l'ouest, sur les côtés du bâtiment de scène. Le passage d'une forme circulaire à un tracé rectiligne se produit à proximité des deux entrées principales, construites selon une géométrie rectiligne et orthogonale.

L'aspect final du théâtre présente donc une forme homogène dont les structures ne sont pas parfaitement symétriques. Cette imperfection dans l'axialité du système résulte de la volonté du concepteur du dernier état du théâtre de réutiliser, dans la mesure du possible, les structures les plus importantes des états précédents comme l'arène et les entrées, tout en donnant au monument une extension maximale.

\subsection{Le choix de l'implantation}

Divers éléments ont déterminé l'implantation du théâtre: sa position aux limites de l'agglomération urbaine, sa relation directe avec les voies de circulation et d'accès à la ville, la proximité de la Seine, ainsi que l'opportunité offerte par l'orographie locale constituée au nord et au centre par une zone plane et au sud par la colline du Toupin.

Cependant, les structures de soutènement adaptent la pente naturelle de la colline orientée est-ouest aux besoins de l'édifice de spectacle. Une nouvelle orientation idéale est créée par l'axe cavea-arène-édifice de scène, de direction sud-nord. La façade postérieure de la scène ou postscaenium 
est tournée vers la ville. Les entrées principales sont situées de part et d'autre de la scène, dans le secteur nord du complexe. En revanche, les entrées situées le long du corridor semi-circulaire qui entoure la cavea sont ouvertes naturellement vers l'extérieur du centre urbain.

\subsection{Dimensions de l'édifice}

D’après les mesures prises à partir de l'extérieur du mur périmétral, et à l'exception des contreforts, les dimensions générales de l'édifice de spectacle de Lillebonne sont de 106,50 $\mathrm{m}$ le long du grand axe est-ouest, soit 360 pieds (3 actus).

Les mesures du petit axe sud-nord ne peuvent pas encore être connues avec précision, en l'absence des mesures exactes du bâtiment de scène, non fouillé. Cependant, les sondages et les observations faites par le passé dans cette partie du théâtre permettent de proposer une mesure de $94,70 \mathrm{~m}$, soit 320 pieds.

Les dimensions de l'arène sont pour le petit axe sud-nord de $35,50 \mathrm{~m}$ environ, soit 120 pieds ou 1 actus. Pour le grand axe est-ouest, on peut restituer une mesure de $47,30 \mathrm{~m}$, soit 160 pieds.

Les axes de l'arène et les axes du théâtre présenteraient donc les rapports suivants: le petit axe de l'arène égale un tiers du grand axe du théâtre; le grand axe de l'arène égale la moitié du petit axe du théâtre.

\subsection{La cavea}

La conque des gradins s'appuie contre la pente naturelle sur environ la moitié de son extension totale, dans l'arc de cercle compris entre le sud-est et le sud-ouest. A cet endroit, deux solutions différentes ont été adoptées pour le soutien des gradins en rapport avec la morphologie du terrain. L'ima et la media cavea sont directement adossées à la déclivité de la colline, modifiée en prévision des exigences des charges à supporter. La summa cavea est construite avec des murs et des remblais (aggestus) qui se fondent sur le sommet de la colline et en prolongent la pente artificiellement.

La seconde partie de la cavea est réalisée, comme l'arène, sur un fond de vallée plane. Elle est soutenue exclusivement par des maçonneries et des remblais. Les vestiges du mur à l'extrémité supérieure de la cavea présentent une hauteur de $19 \mathrm{~m}$ par rapport au niveau de sol de l'arène. La 
hauteur maximale d'origine devait dépasser de quelques mètres la hauteur actuelle des murs. Le monument était complété par une porticus in summa cavea qui couvrait le corridor annulaire.

L'implantation de l'édifice de scène au nord est un élément déterminant de la conception du monument de spectacle qui permet de créer des conditions idéales d'éclairage solaire.

\subsection{Le bâtiment de scène}

Le bâtiment de scène situé sous la route départementale n'a jamais été fouillé. Depuis la limite de l'arène, la scène semble s'étendre au nord sur environ 13 m, soit l'équivalent du tiers de la place Félix Faure. Des éléments appartenant à ces structures ont été mis au jour à plusieurs occasions.

Dès 1826, à la suite des découvertes réalisées dans le terrain de Timothée Holley, Emmanuel Gaillard écrit:

Jusqu'ici on s'était imaginé que l'enceinte théâtrale ne dépassait guère l'enclos acheté par le département. Maintenant il est démontré que l'enceinte était beaucoup plus grande qu'on ne l'avait cru d'abord, qu'elle franchissait la route et la rangée de peupliers qui est au delà, qu'enfin elle se terminait contre la colline qui fait face à celle où étaient assis les spectateurs.

En 1831, Eloi Pigné regrette également que la route n'ait pas été reportée encore plus au nord, à l'emplacement de l'ancienne rue de la Roquette, "car il est probable qu'elle couvre encore une partie notable du théâtre qui suivant l'apparence s'étend jusqu'au castellum».

La présence du bâtiment de scène est identifiée lors des sondages réalisés de 1935 à 1937 sous le contrôle de Paul Franchette et dirigés par Albert Grenier. Celui-ci résume ainsi les structures découvertes sous la place Félix Faure:

Plusieurs galeries, [...] poussées jusqu'à une distance de $12 \mathrm{~m}$, n'ont rencontré que l'assise d'une construction en grand appareil qui pourrait être la fondation de quelque mur du fond de la scène, le premier mur, celui dont on aperçoit le départ, représentant le pulpitum. 
Ces éléments permettent de reconnaître, en face de la cavea, une scène de théâtre, établie sur une partie de l'arène... ${ }^{17}$

En 1962, Maurice Yvart en repère d'autres éléments, complémentaires aux précédents. La rédaction de la Revue des Sociétés savantes de HauteNormandie annonce:

Nous pouvons logiquement penser dorénavant que le théâtre de Lillebonne possède encore à peu près complet son mur de fond de scène derrière le talus de soutènement de la route ${ }^{18}$.

Enfin, en 1986, d'autres éléments de structures ont été découverts lors de travaux consécutifs à la rupture d'un collecteur pluvial longeant la route départementale ${ }^{19}$.

La fouille complète du bâtiment de scène représente actuellement le travail le plus important encore à réaliser dans le monument pour comprendre définitivement la typologie et l'évolution de ses structures.

\section{Nouvelles études et nouvelles fouilles au XXI siècle}

Cependant au fil des années, les intempéries, le développement de la végétation, l'usure du temps ainsi que des interventions de restauration parfois mal adaptées ont rendu les structures dangereuses. Sa fermeture au public est alors devenue indispensable.

Pour remédier à cette situation, un programme d'étude en vue de sa restauration et de sa mise en valeur a été initié par le Conseil général de Seine-Maritime à partir de 2001 : étude préliminaire de l'état sanitaire du monument ${ }^{20}$, relevé topographique précis des structures, étude documentaire des sources d'archives décrivant l'ensemble des découvertes et des travaux intervenus sur le site des origines à l'an $2000^{21}$.

Ces différentes études ont donné lieu à une commande auprès de Régis Martin, architecte en chef des Monuments historiques, en vue de

I7. A. Grenier, «Le Théâtre de Lillebonne», p. 10.

I8. M. Yvart, "Théâtre romain de Lillebonne», p. 13.

19. E. Follain, Rapport sur la fouille et la surveillance de travaux effectués place Félix Faure et rue Victor Hugo à Lillebonne.

20. V. Mutarelli, Le Théâtre romain de Lillebonne. Etude préliminaire.

2. V. Mutarelli, Le Théâtre romain de Lillebonne. Historique des fouilles. 
la restauration, de la mise en sécurité et de l'aménagement du théâtre romain de Lillebonne. Le projet prévoit une campagne de travaux en deux phases successives qui permettront la réouverture progressive du monument au public.

Dans la suite de ce programme d'études préliminaires, la nouvelle campagne de fouilles de 2007-2008 avait pour but de vérifier sur le terrain les résultats de la recherche documentaire, de mesurer l'état des destructions dues aux interventions successives, d'identifier les informations subsistantes essentielles à la compréhension du monument et apporter ainsi des réponses attendues, enfin d'orienter le programme de restauration dans le respect des structures antiques pour une mise en valeur pérenne.

L'étendue de l'emprise de la zone de fouilles a été déterminée par la volonté de lier la recherche historique et archéologique au projet de valorisation, dont la première étape prévoit la construction d'un nouvel accès au monument depuis la place Félix Faure, au niveau de l'escalier E1 et de la partie nord-est de la galerie périphérique. Ce projet vise à ouvrir le site du théâtre vers la ville afin d'améliorer la relation entre le centre-ville et le monument antique, en définissant un nouveau rapport urbanistique qui donnera une plus grande importance aux vestiges archéologiques.

Le secteur concerné par cette campagne de fouilles comprend donc, à l'est du monument, la galerie périphérique depuis son extrémité nord en bordure de la place Félix Faure jusqu'au vomitorium V2, y compris l'entrée axiale est, la zone extérieure au théâtre entre ces deux extrêmes et la cage de l'escalier est donnant accès à la partie supérieure de la cavea, parallèle à la galerie périphérique.

4.1. Le mur de fermeture de l'accès nord-est à la galerie périphérique

Dans la galerie périphérique, sous le mur de clôture actuel du théâtre au nord, une seconde structure d'époque moderne, non référencée dans les sources, a été mise au jour. Il s'agit d'un mur de soutènement de la route départementale édifié le long de la place Félix Faure.

Ce mur a été construit en 1827 aux limites de la place afin de permettre à Emmanuel Gaillard de procéder au déblaiement de la galerie périphérique nord sans risque d'éboulement.

Construit en creusant une tranchée depuis le niveau de la place, le mur de soutènement a été directement fondé sur les pierres en grand 
Fig. 10 — Mur de fermeture nord-est de la galerie périphérique.

appareil d'une structure antique qui murait l'accès nord de la galerie périphérique, en en détruisant une partie: certains de ses éléments sont réutilisés dans les fondations du mur de soutènement. Cette structure, identique à celles qui obstruaient toutes les entrées du théâtre, est constituée de grands blocs en pierre calcaire à rognons de silex encore en place et en bon état de conservation, au moins pour la partie qui en reste (fig. 10). Sa construction intervient à la fin des chantiers de démolition et de récupération des structures du monument qui ont entraîné une modification complète de l'aspect originel du théâtre. Elle est contemporaine de la nouvelle utilisation de l'édifice qui commence au dernier quart du III ${ }^{\mathrm{e}}$ siècle de notre ère.

La fouille a par ailleurs permis de constater que le mur périmétral du monument au nord-est continue au-delà de la limite de la clôture, sous la place Félix Faure.

\subsection{Fondations des murs de la galerie périphérique nord-est}

Les murs de la galerie périphérique nord-est présentent une forme rectiligne, contrairement au reste du théâtre où ils sont concentriques. Les bases de leurs fondations, parallèles et symétriques, sont situées à deux 
niveaux différents. Elles ont été l'une et l'autre construites à fond perdu, mais l'on observe que les fondations du mur intérieur sont situées plus bas que celles du mur périmétral. Le mur interne de la galerie repose en effet sur des maçonneries qui appartiennent au deuxième état du monument.

Les données stratigraphiques des fouilles et l'analyse des maçonneries révèlent que la galerie périphérique a été créée lors de la construction du dernier état du monument, comme le confirment les fouilles du dispositif d'entrée du vomitorium V2 situé plus au sud, dans la partie circulaire de la galerie périphérique.

Si les fondations des murs intérieur et extérieur de la galerie périphérique nord-est sont situées à des niveaux différents, en élévation ces structures présentent un ressaut en retrait en glacis à un niveau identique dans l'ensemble de la galerie nord. Cette symétrie dans l'élévation met en évidence une harmonisation des différences structurelles entre les murs appartenant respectivement au deuxième et au troisième état de l'édifice. A partir du niveau du sol, le corridor nord présentait donc un aspect symétrique et homogène.

Une observation attentive des parements de ses murs en opus vittatum mixtum a permis de retrouver le niveau du pavimentum de la galerie, composé de dalles calcaires qui ont été récupérées lors de l'abandon $\mathrm{du}$ monument, mais dont certaines sont encore en place dans la galerie ouest et dans les podia.

\subsection{Les structures de l'entrée axiale est}

A l'entrée principale est de la galerie périphérique, le mur de soutènement du seuil a été de nouveau mis au jour, ainsi que six éléments de fondation en pierre de calcaire coquillier en grand appareil, assemblés à sec et liés entre eux par des crampons ou agrafes scellés au plomb. Ils constituent les composants architecturaux et décoratifs les plus importants dans ce secteur du monument.

A l'endroit où la morphologie du terrain se modifie en passant de la colline en forte pente à un terrain plat, le monument présente un dispositif d'accès dont la composition est très élaborée. Il est constitué de deux entrées à la galerie périphérique : l'entrée principale fait face au couloir axial est qui conduit à l'arène, tandis qu'à un niveau supérieur, plus au sud, une entrée de dimensions plus réduites donne accès au premier podium par le vomitorium V1 (fig. 11). 
Fig. 11 - Vue d'ensemble de l'entrée est: entrée axiale et entrée au vomitorium V1.

Ces structures situées à l'extrémité et aux points d'entrecroisement des murs contenaient au niveau des fondations les poussées qui descendaient vers le bas depuis les structures de la galerie périphérique supérieure. Elles les transmettaient aux structures fondées sur le terrain uniforme et plat de la galerie périphérique nord, qui faisaient donc ici également fonction de murs-boutants. Au niveau supérieur, elles soutenaient les charges et les poussées des voûtes croisées de la galerie périphérique et les structures de la porticus in summa cavea. Elles supportaient les arcades en grand appareil de l'entrée principale et des accès au couloir axial est et au vomitorium $\mathrm{V} 1$.

L'architecture du dispositif d'entrée est montre donc des caractéristiques spécifiques, sans aucun doute présentes à l'identique à l'entrée ouest encore à étudier, mais qui sont absentes dans les autres accès de la galerie périphérique.

La composition en plan des éléments en grand appareil indique l'existence d'une façade monumentale. L'ouverture, constituée par un chambranle en forme de pilier qui supporte vraisemblablement un arc, est flanquée de deux piliers ou colonnes engagés reposant sur un piédestal.

A l'intérieur de la galerie, au niveau du passage axial vers l'arène, les structures en grand appareil présentent quatre éléments en saillie, 
symétriques, qui permettent de restituer quatre supports verticaux, colonnes ou piliers.

Deux autres bases sont situées à l'intérieur du passage axial, en saillie par rapport aux alignements des parements des murs du couloir de l'entrée axiale est: elles constituent les fondations de deux supports verticaux engagés soutenant probablement un arc en pierre de taille qui annonce la voûte maçonnée du couloir axial vers l'arène.

Aucun élément de décor sculpté n’a été retrouvé dans le secteur de l'entrée est. Cependant, un bloc de corniche modillonnaire et une clef d'arc d'ordre corinthien, retrouvés à l'entrée ouest en 1912, lors des fouilles de Léon de Vesly, offrent des arguments pour une hypothèse de restitution de l'ordre architectural.

\subsection{L'entrée A2 à la galerie et le vomitorium V2}

Les fouilles ont mis au jour le système d'accès au monument au niveau de l'entrée dans la galerie périphérique qui fait face au vomitorium V2, appartenant chronologiquement au dernier état de construction de l'édifice. Il conserve encore un aspect assez proche de celui qu'il avait à l'origine: l'accès au monument se faisait depuis un niveau situé en contrebas de la galerie à cet endroit.

Un couloir a été taillé dans la roche naturelle, dans l'alignement des murs du vomitorium V2. Sa forme s'évase vers l'extérieur pour ouvrir le passage et faciliter ainsi l'entrée et la sortie du public du vomitorium V2 et le canaliser. Un palier et des marches également taillés dans la roche permettaient de monter progressivement jusqu'à l'entrée (fig. 12).

A l'intérieur de la galerie périphérique et du vomitorium V2 ont été découvertes des structures appartenant au deuxième état du théâtre.

Les données stratigraphiques mettent en évidence le fait que la galerie périphérique a été réalisée lors de la construction du dernier état du monument, les couches du chantier de construction du dernier état étant superposées aux couches des chantiers de construction puis de récupération de l'état précédent. Le mur concentrique intérieur de la galerie est directement fondé sur le mur périmétral du deuxième état du théâtre préalablement démoli et récupéré.

Le vomitorium $\mathrm{V} 2$ faisait déjà partie du système d'accès de l'édifice du deuxième état et correspondait à l'une des entrées depuis l'extérieur du monument, avec une légère différence d'orientation. Les fouilles ont mis 
Fig. 12 - Chemin d'accès à l'entrée vers le vomitorium V2.

au jour le mur de soutènement du seuil de l'entrée du vomitorium V2 du deuxième état et le niveau du pavimentum de ce vomitorium, ainsi que deux contreforts placés en fondation des contreforts du troisième état, de part et d'autre de l'entrée du vomitorium (fig. 13).

De nombreux éléments de l'utilisation du monument après son abandon comme édifice de spectacle ont été découverts au début du vomitorium $\mathrm{V} 2$, en particulier de nombreuses séquences de foyers. Le mobilier retrouvé permet de dater cette occupation entre la fin du III ${ }^{\mathrm{e}}$ siècle et la première moitié $\mathrm{du} \mathrm{V}^{\mathrm{e}}$ siècle.

Les parties du monument qui présentaient une couverture étaient la galerie périphérique et les vomitoria. La galerie était couverte par la porticus in summa cavea et les vomitoria par la cavea. Les documents d'archives témoignent de la présence d'habitations dans la galerie périphérique et dans les vomitoria, dont les traces ont été identifiées au cours des fouilles des XIX et $\mathrm{XX}^{\mathrm{e}}$ siècles. 
Fig. 13 - Les deux derniers états du théâtre à l'entrée du vomitorium V2: mur périphérique et contrefort du deuxième état en fondation du mur du vomitorium $\mathrm{V} 2 \mathrm{du}$ troisième état.

\section{Conclusions}

\subsection{Les états du théâtre}

Dans l'état actuel des recherches, le premier état du monument est représenté par les murs circulaires situés à l'intérieur de l'arène, découverts dans les premières années du XX $\mathrm{X}^{\mathrm{e}}$ siècle, fouillés par Léon de Vesly, puis par Paul Franchette. Ces murs, démolis pour la construction de l'arène, ont été fouillés seulement à proximité du premier podium et sont actuellement recouverts d'une couche d'herbe superficielle.

L'arène du monument n'a pas encore été fouillée de manière complète et pourrait contenir d'autres murs circulaires du premier état de l'édifice. La documentation photographique qui illustre les travaux de Paul Franchette en 1938 permet d'observer qu'un second mur circulaire est présent sous le premier podium de l'arène (murs M3, M5 et M1) ${ }^{22}$.

22. P. Franchette, Rapport sur les fouilles archéologiques exécutées en 1938. 
Les structures mises au jour au niveau de l'entrée du vomitorium V2 nous renseignent sur l'évolution de la typologie du monument entre son deuxième et son troisième état: la cavea du deuxième état s'arrêtait au niveau du mur concentrique intérieur du troisième état. Pour agrandir ce deuxième état, on lui a ajouté une galerie périphérique couverte par le sommet de la cavea rehaussée et la porticus in summa cavea, ainsi qu'un deuxième podium. A l'entrée du vomitorium V2, les structures concentriques intérieures de la galerie périphérique du troisième état s'appuient sur les structures du mur extérieur de la cavea du deuxième état.

Les informations issues du programme de fouilles apportent un début de résultat d'un grand intérêt pour la datation et l'étude de la typologie des deuxième et troisième états de construction du monument, mais elles demandent à être complétées et confirmées, l'étendue des surfaces fouillées qui les contenaient étant très limitée. Ces premières indications sont cependant essentielles pour la suite des recherches archéologiques.

La découverte de l'obstruction de l'accès nord-est à la galerie périphérique qui ouvrait vers le centre urbain est un élément nouveau qui indique que le monument fut alors totalement isolé de son environnement.

En effet, à la suite de l'abandon du monument comme édifice de spectacle, ce sont tous les accès extérieurs vers la galerie périphérique qui ont été fermés par des structures en blocs de pierre calcaire d'importantes dimensions, isolant et fortifiant ainsi l'édifice pendant la période du BasEmpire.

Cette information ouvre une nouvelle problématique concernant le système d'accès à l'édifice après son changement d'utilisation et les modifications de ses caractéristiques architecturales.

Un seul alignement des blocs qui fermaient la galerie périphérique au nord a été fouillé. Une partie de cette structure étant située sous la route départementale, son exploration complète devra s'inscrire dans le cadre d'un programme de fouilles qui intéressera tous les vestiges du théâtre encore situés dans le sous-sol de la place Félix Faure.

\subsection{Le rempart du Bas-Empire}

Plusieurs hypothèses envisageaient l'existence d'une structure appartenant au mur d'enceinte du Bas-Empire qui aurait ceinturé le pourtour du mur périmétral du théâtre. Ces hypothèses auraient été confortées par la 
mise au jour en 1836, à l'ouest de l'édifice, adossée au contrefort 68, d'une structure dont la typologie semblait conforme à celle des remparts du Bas-Empire ${ }^{23}$.

Les fondations décrites par Achille Deville en 1836 appartiennent à une structure extérieure qui se trouvait en contact sans être liaisonnée avec le contrefort 68, donc sans rapport structurel direct avec le corps même du mur périmétral. Partant du parement extérieur du contrefort, la structure suivait une courbe en direction du centre urbain actuel, vers le nord-ouest, sur une longueur de 5 m environ.

Les résultats de la récente campagne de fouilles et le nettoyage en cours du mur périmétral conduisent à exclure la présence d'un rempart du Bas-Empire qui aurait encerclé le monument. Aucune structure d'une telle importance n’a été observée à l'extérieur du mur périmétral.

Après l'abandon du monument comme édifice de spectacle, toutes les ouvertures ont été obstruées par des murs constitués de grands blocs de réemploi en pierre calcaire assemblés à sec. L'édification de ces structures présentant une grande qualité de résistance et de durabilité exprime la volonté d'isoler et de fortifier le monument.

L'épaisseur du mur extérieur d'environ $2 \mathrm{~m}$ à laquelle s'ajoutait la largeur des contreforts, de 0,90 à $3 \mathrm{~m}$ et l'imposante hauteur de l'édifice au niveau de la porticus in summa cavea, sans doute proche de $30 \mathrm{~m}$ depuis les deux entrées axiales à l'arène, rendaient tout à fait superflue la construction d'un rempart extérieur qui aurait doublé le mur périmétral.

Si le théâtre a été intégré au système de défense de la ville, cela s'est donc fait par la fortification du mur périmétral du théâtre lui-même.

En conclusion, le rapport du théâtre avec le mur d'enceinte de la ville conduit à envisager deux systèmes fortifiés différents et exclusifs l'un de l'autre:

- soit deux ensembles défensifs constitués d'une part par le rempart du Bas-Empire de la ville, retrouvé pendant les fouilles de Timothée Holley en 1824, fondé sur la chaussée extérieure qui longeait le mur du postscaenium du théâtre, d'autre part par la fortification du théâtre lui-même, séparé du rempart de la ville dont il constituait un avant-poste isolé;

- soit un seul ensemble défensif intégrant les deux structures dans le même système fortifié, par une liaison placée ou aux extrémités

23. A. Deville, Notes sur les sculptures et les mosaïques découvertes à Lillebonne en 1836. 
du mur périmétral au nord-ouest et au nord-est, ou au niveau du bâtiment de scène.

Seule la fouille des vestiges encore ensevelis sous l'actuelle place Félix Faure pourra apporter des réponses à cette problématique à la fois historique et archéologique.

Vincenzo Mutarelli

Service archéologique de Seine-Maritime 


\section{BIBLIOGRAPHIE}

\section{Sources manuscrites}

Deville, Achille, Notes sur les sculptures et les mosä̈ques découvertes à Lillebonne en 1836, AD76, 4T263.

Estaintot, Robert Langlois, vicomte d', Procès verbaux de la Commission des Antiquités de la Seine-Inférieure, 10 décembre 1880.

Franchette, Paul, Rapport sur les fouilles archéologiques exécutées de 1935 à 1937 au théatre antique de Lillebonne..., Rouen, le 20 septembre 1937, AD76, 4T166.

-, Rapport sur les fouilles archéologiques exécutées en 1938, Rouen, le 15 octobre 1938, AD76, 4T166.

Gaillard, Emmanuel, Trois dissertations sur diverses parties du théatre romain de Lillebonne, 10 janvier 1830, Manuscrit, AD 76, 4T165.

Guyoт, André, dossiers "Lillebonne, travaux et découvertes", 19081915, Archives du Musée des Antiquités de la Seine-Maritime.

Pigné, Eloi, Panorama de Lillebonne. $4^{\mathrm{e}}$ partie, Archéologie ou Notice sur les antiquités de Lillebonne, Lillebonne, 1831, Manuscrit.

\section{Sources publiées}

Caylus, Anne Claude Philippe de Thubières, comte de, Recueil d'Antiquités égyptiennes, étrusques, grecques, romaines et gauloises, Paris, Desaint et Saillant, 1764, t. VI.

Cochet, Abbé Jean Benoît Désiré, La Normandie souterraine, Paris, Derache, 1855.

Cronicae Fontanellensis Abbatiae, in Neustria Pia seu de omnibus et singulis abbatiis et prioratibus totius Normaniae, éd. A. Du Monstier, Rouen, Jean Berthelin, 1663. 
Deville, Achille, "Antiquités, pierres sculptées découvertes à Lillebonne en 1836 ", dans Précis de l'Académie de Rouen, 1837.

Duplessis, Dom Toussaint, Description géographique et historique de la Haute-Normandie, Paris, Didot, 1740.

Mazois, François, Les Ruines de Pompéi, Paris, Didot, 1812-1838.

—, "Considérations sur la forme et la distribution des théâtres antiques", dans Théatre complet des Latins. Augmenté de dissertations, etc., J.-B. Levée et l'Abbé Le Monnier, Paris, A. Chasseriau, 1820, t. I, p. XLIX-LXVI.

Rever, François, Mémoire sur les ruines de Lillebonne, arrondissement du Havre, Seine-Inférieure, Rouen (Evreux), Ancelle, 1821 [1825].

\section{Etudes}

Follain, Eric, Rapport sur la fouille et la surveillance de travaux effectués place Félix Faure et rue Victor Hugo à Lillebonne, Rouen, SRA, 1986, document final de synthèse, 1986, no 92.

Grenier, Albert, «Le Théâtre de Lillebonne», Revue des Sociétés savantes de Haute-Normandie, 2 (1956), p. 7-12.

-, Manuel d'archéologie préhistorique, celtique et gallo-romaine. Archéologie gallo-romaine, volume III, $3^{\mathrm{e}}$ partie, L'architecture.

2. Ludi et circenses, théâtres, amphithéatres, cirques, Paris, Picard, 1958.

Mutarelli, Vincenzo, Le Théâtre romain de Lillebonne. Etude préliminaire, Rouen, Conseil général de Seine-Maritime, SRA, 2001.

—, Le Théâtre romain de Lillebonne. Historique des fouilles, Rouen, Conseil général de Seine-Maritime, SRA, 2006.

Yvart, Maurice, "Théâtre romain de Lillebonne. Campagne de restauration 1961-1962, Addenda", Revue des Sociétés savantes de HauteNormandie, Archéologie-Préhistoire, 26 (1962), p. 3-16.

\section{Crédits iconographiques}

Fig. 1 :

Cliché Studio A. Huon, Caudebec-en-Caux. 
Fig. 2-3, 5-6 et 10-13:

Clichés Vincenzo Mutarelli.

Fig. 4:

Cliché Yohan Deslandes.

Fig. 7 :

Cliché Musée municipal de Lillebonne.

Fig. 8, 8bis et 9:

Grenier, Albert, "Le théâtre de Lillebonne», Revue des Sociétés savantes de Haute-Normandie, 2 (1956), p. 7-12. 
